DESY09-161

hep-th/yymmnn

October 2009

\title{
Initial time singularities and admissible initial states for a system of coupled scalar fields
}

\author{
Jürgen Baacke1 \\ Fakultät Physik, Technische Universität Dortmund \\ D - 44221 Dortmund, Germany \\ and \\ Nina Kevlishvili: \\ Deutsches Elektronen Synchrotron DESY \\ D - 22603 Hamburg, Germany, \\ Andronikashvili Institute of Physics, GAS \\ 0177 Tbilisi, Georgia
}

\begin{abstract}
We discuss the problem of initial states for a system of coupled scalar fields out of equilibrium in the one-loop approximation. The fields consist of classical background fields, taken constant in space, and quantum fluctuations. If the initial state is the adiabatic vacuum, i.e., the ground state of a Fock space of particle excitations that diagonalize the mass matrix, the energy-momentum tensor is infinite at $t=0$, its most singular part behaves as $1 / t$. When the system is coupled to gravity this presents a problem that we solve by a Bogoliubov transformation of the naive initial state. As a side result we also discuss the canonical formalism and the adiabatic particle number for such a system. Most of the formalism is presented for Minkowksi space. Embedding the system and its dynamics into a flat FRW universe is straightforward and we briefly address the essential modifications.
\end{abstract}

\footnotetext{
${ }^{1}$ e-mail: juergen.baacke@tu-dortmund.de

${ }^{2}$ e-mail: nina.kevlishvili@desy.de
} 


\section{Introduction}

The question of initial states in nonequilibrium quantum field theory has found considerable interest recently [1, 2, 3, 4, 5, 6, 17, 8, 9, 10, 11, 12, on various grounds. As a very practical aspect it was realized in numerical simulations of quantum fields in cosmology [13, 2, 14], that the energy-momentum tensor had initial time singularities if the initial state was taken as the naive Fock-space vacuum. These had to be removed when coupling the field to gravity, e.g. in a Friedmann universe. A more speculative aspect that has attracted some interest recently was the question, whether the choice of initial state can be expected to leave an imprint in the CMB spectrum. In any case it is a question of principle, to what extent the choice of initial state is constrained in an interacting theory of particles by consistency requirements.

As a general aspect of quantum field theory, the problem of initial conditions was realized long ago. It ultimately can traced back to the fact that one switches on the interaction at some time $t=0$. In the case of nonequilibrium dynamics we have to impose initial conditions for the background fields. In most applications the initial state was taken to be the adiabatic vacuum, which would be the ground state if all background fields were held fixed forever. When one starts to evolve the system dynamically at least the second derivative of the fields will be discontinuous on account of the second order differential equation, which for $t>0$ determines their evolution. In cosmology it is already the first derivative of the scale parameter which for $t>0$ is determined by the Friedmann equation. Such singularities have been noted for the first time by Stückelberg [15], they are discussed briefly in the textbook of Bogoliubov and Shirkov [16. The phenomenon has been identified as a kind of 'Casimir effect' connected to the initial time surface by Symanzik [17]. In the context of quantum field theory out of equilibrium the presence of such singularities has been noticed by various authors.

The solution proposed by Symanzik is the introduction of surface counterterms in addition to the usual "bulk" counterterms of perturbative quantum field theory. In the context of nonequilibrium quantum field theory this line has been pursued in Refs. [9, 10].

The introduction of initial time surfaces singles out the particular time at which one starts the evolution to the extent that the surface counterterms become part of the field theory for $t>0$. The approach of modifying the

naive initial vacuum state [18, 2, 8] seems to be more pragmatic; the idea is to find the minimal requirements on an initial state that could arise from 
a previous dynamical evolution. The latter aspect is discussed in [19]. The technique used for constructing such an initial state consists of finding a Bogoliubov transformation of the naive adiabatic vacuum. For the singlechannel case and scalar fluctuations this has been done in Ref. [2] and for fermion fluctuations in Ref. [20]. These results were used in Refs. [21, 14] in formulating the renormalized equations in a flat FRW universe.

Our approach is based on a mode function formalism that has been introduced, for coupled channels, in Ref. [22]. The formalism ensures the conservation of energy with one-loop or Hartree quantum backreaction and has been renormalized along the lines of Ref. [23]. There the initial state was chosen to be the adiabatic vacuum based on a Fock space of particle excitations that diagonalize the initial mass matrix. It is this initial state that we will improve here. In Ref. [22] renormalization is based on a perturbative expansion close to standard perturbation theory, in the same way as in Ref. [23]. The same expansion was used in Ref. [2] for analyzing the initial time singularity. This analysis can be carried over in a straightforward way to the case of coupled fields. Most other analyses of the singular early time behavior were based on the eikonal expansion. An eikonal formalism for coupled systems has been formulated recently [24]. We are not aware, however, of an eikonal expansion for coupled-channel systems.

The quantum expansion of the fluctuation fields is formulated in such a way that the canonical commutation relations hold for $t=0$. It was not discussed in Ref. [22] how they continue to hold for $t>0$. Though this is to be expected it is not entirely obvious, and in fact leads some nontrivial relations for the fluctuation modes which prove to be useful for our formalism. This is discussed in Appendix B. Another approach to the canonical formalism for coupled-channel systems was introduced in Ref. [24], and this is another reason for verifying that the scheme of Ref. [22] works correctly.

Though our main subject here is the choice of the initial state, with hindsight of coupling the system to gravity, we take the occasion for discussing the concept of adiabatic particle number within our formalism. This is suggested by the fact that we have to discuss Bogoliubov transformations for coupled systems anyway and that particle numbers are usually defined by the coefficients of these transformations.

The paper is organized as follows: In Sec. 2 we introduce the model we want to consider, a system of two coupled quantum fields with masses and a general fourth order potential. We define the decomposition into classical and fluctuation fields and the evolution of the fluctuations. In Sec. 3 we 
discuss the behavior of the Green's function at early times and construct a Bogoliubov transformation in order to reduce the leading singular behavior such that the leading time derivatives become finite at the intial time. In Sec. 4 we present the expectation value of the energy-momentum tensor in the Bogoliubov-transformed initial state. In Sec. 5 we discuss the concept of adiabatic particle number. Some more technical subjects are transferred to the Appendices: the Bogoliubov transformation for coupled systems in Appendix $\mathrm{A}$ and some aspects of the canonical formalism in Appendix B.

\section{The model}

We consider a system of two coupled scalar quantum fields with a Lagrangian density of the form

$$
\mathcal{L}=\left[\frac{1}{2} \partial_{\mu} \phi_{i} \partial^{\mu} \phi_{i}+\frac{1}{2} m_{i}^{2} \phi_{i}^{2}\right]+\frac{\lambda_{i j}}{4} \phi_{i}^{2} \phi_{j}^{2}
$$

where the indices $i, j$ take the values 1 and 2 . The hybrid model of inflation [25, 26, 27] with

$$
\mathcal{L}=\frac{1}{2} \partial_{\mu} \phi \partial^{\mu} \phi+\frac{1}{2} \partial_{\mu} \chi \partial^{\mu} \chi+\frac{1}{2} m^{2} \phi^{2}+\frac{\alpha}{4}\left(\chi^{2}-v^{2}\right)^{2}+\frac{\lambda}{2} \phi^{2} \chi^{2}
$$

is of this form with $\lambda_{11}=0, \lambda_{12}=\lambda, \lambda_{22}=\alpha, m_{1}^{2}=m^{2}$ and $m_{2}^{2}=-\alpha v^{2}$. Also some models involving supersymmetric flat directions [28, 29] are of this type. The generalization to a general mass matrix and a general fourth order potential is possible, but we do not want do overburden the formalism with a profusion of indices. Also, the limitation to two fields is not essential.

We separate the fields $\phi$ into classical fields and fluctuations via

$$
\phi_{i}=\varphi_{i}+\psi_{i}
$$

The classical Lagrangian density then retains the form

$$
\mathcal{L}^{(0)}=\left[\frac{1}{2} \partial_{\mu} \varphi_{i} \partial^{\mu} \varphi_{i}+\frac{1}{2} m_{i}^{2} \varphi_{i}^{2}\right]+\frac{\lambda_{i j}}{4} \varphi_{i}^{2} \varphi_{j}^{2},
$$

while the fluctuation Lagrangian, of second order in the fluctuations, becomes

$$
\mathcal{L}^{(2)}=\left[\frac{1}{2} \partial_{\mu} \psi_{i} \partial^{\mu} \psi_{i}+\frac{1}{2} m_{i}^{2} \psi_{i}^{2}\right]+\frac{\lambda_{i j}}{2}\left[\varphi_{i}^{2} \psi_{j}^{2}+2 \varphi_{i} \varphi_{j} \psi_{i} \psi_{j}\right]
$$


This can be written as

$$
\mathcal{L}^{(2)}=\sum_{i=1}^{2}\left[\frac{1}{2} \partial_{\mu} \psi_{i} \partial^{\mu} \psi_{i}+\frac{1}{2} \mathcal{M}_{i j}^{2}(\varphi) \psi_{i} \psi_{j}\right],
$$

with

$$
\begin{aligned}
& \mathcal{M}_{11}^{2}=m_{1}^{2}+3 \lambda_{11} \varphi_{1}^{2}+\lambda_{12} \varphi_{2}^{2} \\
& \mathcal{M}_{12}^{2}=2 \lambda_{12} \varphi_{1} \varphi_{2} \\
& \mathcal{M}_{22}^{2}=m_{2}^{2}+3 \lambda_{22} \varphi_{2}^{2}+\lambda_{12} \varphi_{1}^{2}
\end{aligned}
$$

If the field is coupled to gravity in a flat FRW universe, the fluctuation mass matrix takes a similar form. After conformal rescaling of fields and momenta (see, e.g., Refs. [30, 13]) one just has to replace

$$
m_{i}^{2} \rightarrow\left[m_{i}^{2}+\left(\xi_{i}-\frac{1}{6}\right) R\right] a^{2} .
$$

Here $a$ is the scale parameter, $R$ the Ricci scalar, and the $\xi_{i}$ are the conformal couplings.

In the following we restrict ourselves to homogeneous background fields $\varphi_{i}(t)$, so the mass matrix depends on time only. In the FRW universe the time parameter is conformal time, and we have an additional time dependence via $a(\tau)$ and $R(\tau)$.

We separate the fluctuation mass matrix into its initial value and a "potential" $\mathcal{V}$ via

$$
\mathcal{M}_{i j}^{2}(t)=\mathcal{M}_{i j}^{2}(0)+\mathcal{V}_{i j}(t) .
$$

We diagonalize the initial mass matrix by

$$
\mathcal{M}_{i j}^{2}(0) f_{j 0}^{\alpha}=m_{\alpha 0}^{2} f_{i 0}^{\alpha} .
$$

The eigenvectors $f_{i 0}^{\alpha}$ are chosen to be real, and normalized to unity:

$$
\sum_{i=1}^{2} f_{i 0}^{\alpha} f_{i 0}^{\beta}=\delta^{\alpha \beta}
$$

The latin subscripts refer to the field components, as before, and the Greek superscripts refer to the two independent solutions of the eigenvalue equation. We now define a set of mode functions $f_{i}^{\alpha}(k, t)$ for homogeneous background 
field in the following way:

(i) their time evolution is determined by

$$
\ddot{f}_{i}^{\alpha}(k, t)+k^{2} f_{i}^{\alpha}(k, t)+\mathcal{M}_{i j}^{2}(t) f_{j}^{\alpha}(k, t)=0 ;
$$

(ii) the initial conditions are specified as

$$
\begin{aligned}
& f_{i}^{\alpha}(k, 0)=f_{i 0}^{\alpha}, \\
& \dot{f}_{i}^{\alpha}(k, 0)=-i \Omega_{\alpha 0} f_{i 0}^{\alpha},
\end{aligned}
$$

where we have introduced the frequencies

$$
\Omega_{\alpha 0}(k)=\sqrt{m_{\alpha 0}^{2}+k^{2}} .
$$

The functions $f_{i}^{\alpha}(k, t)$ form a set of linearly independent solutions of the system of mode equations.

The fields $\psi_{i}(\mathbf{x}, t)$ are quantum fields. For a homogeneous background we can expand them as

$$
\psi_{i}(\mathbf{x}, t)=\sum_{\alpha} \int \frac{d^{3} k}{(2 \pi)^{3} 2 \Omega_{\alpha 0}}\left[a_{\alpha}(\mathbf{k}) f_{i}^{\alpha}(k, t)+a_{\alpha}^{\dagger}(-\mathbf{k}) f_{i}^{\alpha *}(k, t)\right] e^{i \mathbf{k x}} .
$$

The canonical commutation relations are

$$
\left[a_{\alpha}(\mathbf{k}), a_{\beta}^{\dagger}\left(\mathbf{k}^{\prime}\right)\right]=(2 \pi)^{3} 2 \Omega_{\alpha 0}(k) \delta_{\alpha \beta} \delta^{3}\left(\mathbf{k}-\mathbf{k}^{\prime}\right) .
$$

In the following we will need the two-point functions at the coincidence limit, the "fluctuation integrals"

$$
\begin{aligned}
\mathcal{F}_{i j}(t) & =<0\left|\psi_{i}(\mathbf{x}, t) \psi_{j}(\mathbf{x}, t)\right| 0> \\
& =\sum_{\alpha} \int \frac{d^{3} k}{(2 \pi)^{3} 2 \Omega_{\alpha 0}(k)} f_{i}^{\alpha}(k, t) f_{j}^{\alpha *}(k, t) .
\end{aligned}
$$

Here the expectation value is taken in the vacuum state of a Fock space, whose quanta have the initial masses $m_{\alpha}(0)$. This is the "adiabatic vacuum", defined by

$$
a_{\alpha}(\mathbf{k}) \mid 0>=0 \quad \forall \alpha, \mathbf{k} .
$$

Of course this is not the ground state of the system, and the creation and annihilation operators $a_{\alpha}^{\dagger}(\mathbf{k})$ and $a_{\alpha}(\mathbf{k})$ do not create free particles with the masses $m_{i}$. We discuss some aspects of the canonical formalism in Appendix $\mathrm{B}$, in particular we establish that the fluctuation integral as defined above is real and symmetric in $i$ and $j$, though this is not apparent on the right hand side of Eq (2.19). 


\section{The initial time singularity of the Green's function and the modified initial state}

The quantum backreaction of the fluctuations onto the classical fields can be derived using the closed-time-path formalism [31, 32]. For the quantum field theories that we consider here, it has been formulated in several seminal publications [33, 34, 35, 18, 36]. We do not repeat this here. If one just considers the one-loop quantum backreaction the relevant equations take a rather intuitive form. The equations of motion for the classical fields become

$$
\begin{aligned}
\ddot{\varphi}_{1}+m_{1}^{2} \varphi_{1} & +\lambda_{11} \varphi_{1}^{3}+\lambda_{12} \varphi_{1} \varphi_{2}^{2} \\
& +3 \lambda_{11} \varphi_{1} \mathcal{F}_{11}+\lambda_{12} \varphi_{1} \mathcal{F}_{22}+2 \lambda_{12} \varphi_{2} \mathcal{F}_{12}=0
\end{aligned}
$$

and an analogous equation for $\varphi_{2}$. As will be analyzed below, the fluctuation integrals $\mathcal{F}_{i j}(t)$ are singular at $t=0$, the time where we start the evolution. As $t \searrow 0$ it behaves as $t \ln t$. Though this represents a mathematical singularity, it is finite and even zero at $t=0$. So it will not prevent us from starting a numerical simulation. The singular behavior becomes a problem when we couple the field to gravity. The dynamics of the FRW scale factor $a$ is determined by the energy-momentum tensor, which involves second time derivatives of the two-point function. If one analyzes the energy-momentum tensor, one indeed finds, near $t=0$, a time dependence of the form $1 / t$ in $T_{\mu}^{\mu}$. This then prevents one from starting the dynamical evolution. Of course, even in flat space this infinity is an undesirable and unphysical feature of the energy-momentum tensor.

As the energy-momentum tensor is a rather involved expression, especially after renormalization, we first consider the fluctuation integral and find a way to remove its initial singularity, such that its first and second time derivatives at $t=0$ become finite. This requires less algebra and, as we have seen previously [2], this is sufficient for making the energy-momentum tensor finite near $t=0$.

The fluctuation integrals are ultraviolet divergent. The divergences can be analyzed [23] by expanding with respect to orders in $\mathcal{V}$ which is equivalent to expanding with respect to the couplings $\lambda_{i j}$. This allows one to remove the divergent parts and the dynamics is determined by the remaining finite parts. A closer analysis shows that, on the level of fluctuation integrals, the

contributions of zeroth and first order in $\mathcal{V}$ are ultraviolet divergent. One 
finds [23], up to first order in $\mathcal{V}$,

$$
\begin{aligned}
& \mathcal{F}_{i j}=\int \frac{d^{3} k}{(2 \pi)^{3}} \sum_{\alpha} \frac{1}{2 \Omega_{\alpha 0}} f_{i}^{\alpha}(t) f_{j}^{\alpha *}(t) \simeq \int \frac{d^{3} k}{(2 \pi)^{3}}\left\{\sum_{\alpha} \frac{1}{2 \Omega_{\alpha 0}} f_{i 0}^{\alpha} f_{j 0}^{\alpha *}\right. \\
& +\sum_{\alpha \beta} \frac{1}{2 \Omega_{\alpha 0} \Omega_{\beta 0}} f_{i 0}^{\alpha} f_{j 0}^{\beta}\left[-\frac{1}{\Omega_{\alpha 0}+\Omega_{\beta 0}}\left(\mathcal{V}_{\alpha \beta}(t)-\mathcal{V}_{\alpha \beta}(0) \cos \left[\left(\Omega_{\alpha 0}+\Omega_{\beta 0}\right) t\right]\right)\right. \\
& +\frac{1}{\left(\Omega_{\alpha 0}+\Omega_{\beta 0}\right)^{2}} \dot{\mathcal{V}}_{\alpha \beta}(0) \sin \left[\left(\Omega_{\alpha 0}+\Omega_{\beta 0}\right) t\right] \\
& +\frac{1}{\left(\Omega_{\alpha 0}+\Omega_{\beta 0}\right)^{3}}\left(\ddot{\mathcal{V}}_{\alpha \beta}(t)-\ddot{\mathcal{V}}_{\alpha \beta}(0) \cos \left[\left(\Omega_{\alpha 0}+\Omega_{\beta 0}\right) t\right]\right) \\
& \left.\left.+\frac{1}{\left(\Omega_{\alpha 0}+\Omega_{\beta 0}\right)^{3}} \int d t^{\prime} \ddot{\mathcal{V}}_{\alpha \beta}\left(t^{\prime}\right) \cos \left[\left(\Omega_{\alpha 0}+\Omega_{\beta 0}\right)\left(t-t^{\prime}\right)\right]\right]\right\}
\end{aligned}
$$

Several integrations by parts have been performed in order to separate the high momentum power behavior. The first term in the integrand is quadratically divergent, and the one proportional to $\mathcal{V}(t)$ is logarithmically divergent. In the process of renormalization these terms are removed and included in the mass and coupling constant renormalizations. The contribution proportional to $\mathcal{V}(0)$ vanishes as $\mathcal{V}(0)=0$ by definition, see Eq. (2.10). The terms proportional to $\dot{\mathcal{V}}(t)$ and $\ddot{\mathcal{V}}(t)$ are finite at all times. The nonanalytic parts are those proportional to $\dot{\mathcal{V}}(0)$ and $\ddot{\mathcal{V}}(0)$. Near $t=0$ we find that the momentum integrals which multiply $\dot{\mathcal{V}}(0)$ and $\ddot{\mathcal{V}}(0)$ behave as

$$
\begin{aligned}
& \int \frac{d^{3} k}{(2 \pi)^{3}} \frac{1}{2 \Omega_{\alpha 0} \Omega_{\beta 0}\left(\Omega_{\alpha 0}+\Omega_{\beta 0}\right)^{2}} \sin \left[\left(\Omega_{\alpha 0}+\Omega_{\beta 0}\right) t\right] \simeq-\frac{1}{8 \pi^{2}} t \ln \left[\left(m_{\alpha}+m_{\beta}\right) t\right], \\
& \int \frac{d^{3} k}{(2 \pi)^{3}} \frac{1}{2 \Omega_{\alpha 0} \Omega_{\beta 0}\left(\Omega_{\alpha 0}+\Omega_{\beta 0}\right)^{3}} \cos \left[\left(\Omega_{\alpha 0}+\Omega_{\beta 0}\right) t\right] \simeq \frac{1}{16 \pi^{2}} t^{2} \ln \left[\left(m_{\alpha}+m_{\beta}\right) t\right] .
\end{aligned}
$$

So in general the first and second derivatives of the fluctuation integrals would be infinite at $t=0$.

As we have mentioned previously there are two methods for getting rid of this singular behavior: either one introduces surface counterterms or one modifies the initial state. Our approach is the second one, and we have formulated this modification of the initial state as a Bogoliubov transformation. The singular behavior is obviously related to the large momentum behavior of the integrand. So the modification of the initial state will constrain only its ultraviolet behavior. We are still free to modify it at finite momenta, or 
with contributions that vanish sufficiently fast at large momenta, as e.g. a thermal initial state.

The Bogoliubov transformation and its consequences for the fluctuation integral are presented in detail in Appendix A. The general concept implies that we replace our naive initial state, the vacuum state for quanta of masses $m_{i 0}$ by a transformed vacuum state, annihilated by a superposition of annihilation operators $a_{\alpha}(\mathbf{k})$ and creation operators $a_{\alpha}^{\dagger}(-\mathbf{k})$. The essential formulae are:

(i) the definition of the transformation

$$
\tilde{a}_{\alpha}(\mathbf{k})=\sum_{\beta} \sqrt{\frac{\Omega_{\alpha 0}}{\Omega_{\beta 0}}}\left[C^{\alpha \beta} a_{\beta}(\mathbf{k})-S^{\alpha \beta} a_{\beta}^{\dagger}(-\mathbf{k})\right] ;
$$

(ii) the definition of a new vacuum state $\mid \tilde{0}>$ via

$$
\tilde{a}_{\gamma}(\mathbf{k})\left|\tilde{0}>=\sqrt{\frac{\Omega_{\gamma 0}}{\Omega_{\alpha 0}}} C^{\gamma \alpha}\left[a_{\alpha}(\mathbf{k})-\sqrt{\frac{\Omega_{\alpha 0}}{\Omega_{\beta 0}}} \rho_{\alpha \beta} a_{\beta}^{\dagger}(-\mathbf{k})\right]\right| \tilde{0}>=0,
$$

where $\rho=C^{-1} S$ is a symmetric matrix;

(iii) the definition of a matrix $M_{\alpha \beta}$ which is introduced via the expectation value of $a_{\alpha}(\mathbf{k}) a_{\beta}^{\dagger}\left(\mathbf{k}^{\prime}\right)$ in the new vacuum:

$$
<\tilde{0}\left|a_{\alpha}(\mathbf{k}) a_{\beta}^{\dagger}\left(\mathbf{k}^{\prime}\right)\right| \tilde{0}>=(2 \pi)^{3} \delta^{3}\left(\mathbf{k}-\mathbf{k}^{\prime}\right) 2 \sqrt{\Omega_{\alpha 0} \Omega_{\beta 0}} M_{\alpha \beta}(k) ;
$$

and

(iv) the relation between $\rho$ and $M$

$$
M-\rho M^{\top} \rho^{\dagger}=I,
$$

which ensures that the commutator of $\tilde{a}_{\alpha}(\mathbf{k})$ and $\tilde{a}_{\beta}^{\dagger}\left(\mathbf{k}^{\prime}\right)$ is canonical. All matrices which we have introduced here depend on $k=|\mathbf{k}|$.

As derived in Appendix A, the fluctuation integral, when evaluated in the Bogoliubov-transformed vacuum, takes the form

$$
\begin{aligned}
& \tilde{\mathcal{F}}_{i j}(t)=<\tilde{0}\left|\psi_{i}(\mathbf{x}, t) \psi_{j}(\mathbf{x}, t)\right| \tilde{0}> \\
= & \frac{1}{2} \int \frac{d^{3} k}{(2 \pi)^{3}} \sum_{\alpha, \beta} \frac{1}{2 \sqrt{\Omega_{\alpha 0} \Omega_{\beta 0}}}\left[f_{i}^{\alpha}(t) f_{j}^{\beta}(t) \rho^{\beta \kappa} M_{\alpha \kappa}\right. \\
& +f_{i}^{\alpha}(t) f_{j}^{\beta *}(t) M_{\alpha \beta} \\
& +f_{i}^{\alpha *}(t) f_{j}^{\beta}(t) \rho^{\alpha \kappa *} \rho^{\beta \lambda} M_{\kappa \lambda} \\
& \left.+f_{i}^{\alpha *}(t) f_{j}^{\beta *}(t) \rho^{\alpha \kappa *} M_{\kappa \beta}\right] .
\end{aligned}
$$


We now have to determine $\rho$ in such a way as to cancel the initial singularities which are contained in the integral over $f_{i}^{\alpha}(t) f_{j}^{\beta *}(t)$. As discussed below Eq. (3.2) the dangerous contributions are those involving $\dot{\mathcal{V}}_{\alpha \beta}(0) \sin \left[\left(\Omega_{\alpha 0}+\Omega_{\beta 0}\right) t\right]$ and $\left.\ddot{\mathcal{V}}_{\alpha \beta}(0) \cos \left[\Omega_{\alpha 0}+\Omega_{\beta 0}\right) t\right]$. They have to be cancelled by the terms proportional to $\rho$ and $\rho^{2}$ generated by the Bogoliubov transformation. If one considers Eqs. (3.6) and (3.7) one realizes that the determination of $\rho$ seems to be marred already by the nonlinear relation between $M$ and $\rho$. We have to realize, however, that there is no unique choice for $\rho$, anyway. All we need is a cancellation of the dangerous terms at large momenta. These contributions are divided, in the integrand, by combinations of $\Omega_{\alpha 0}$ and $\Omega_{\beta 0}$ which asymptotically behave as $k^{-4}$ and $k^{-5}$, respectively. So these terms become small asymptotically, and to get the correct asymptotic behavior of the matrix elements of $\rho$ we can work in the linear approximation. In this approximation we have $M_{\alpha \beta} \simeq \delta_{\alpha \beta}, \rho^{\beta \kappa} M_{\alpha \kappa} \simeq \rho^{\beta \alpha}$, and $M_{\kappa \lambda} \rho^{\alpha \kappa *} \rho^{\beta \lambda} \simeq 0$. Furthermore, we can approximate $f_{i}^{\alpha}(t) \simeq f_{i 0}^{\alpha} \exp \left(-i \Omega_{\alpha 0} t\right)$, whenever it appears multiplied by $\rho$. Corrections would be of order $\rho * \mathcal{V}$; as $\mathcal{V}$ is of order $\rho$ this would be of order $\rho^{2}$.

When rewriting the fluctuation integral in the Bogoliubov-transformed vacuum $\tilde{\mathcal{F}}_{i j}$ we use the approximations we have just mentioned. We further use the expansion of $f_{i}^{\alpha}(t) f_{j}^{\alpha *}(t)$ as it appears in Eq. (3.2), but we remove the renormalization parts. We then obtain, to first order in $\mathcal{V}$ and $\rho$

$$
\begin{aligned}
& \tilde{\mathcal{F}}_{i j} \simeq \int \frac{d^{3} k}{(2 \pi)^{3}}\left\{\sum_{\alpha, \beta} \frac{1}{2 \sqrt{\Omega_{\alpha 0} \Omega_{\beta 0}}} f_{i 0}^{\alpha} f_{j 0}^{\beta}\left[\rho^{\alpha \beta} e^{-i\left(\Omega_{\alpha 0}+\Omega_{\beta 0}\right) t}+\rho^{\alpha \beta *} e^{i\left(\Omega_{\alpha 0}+\Omega_{\beta 0}\right) t}\right]\right. \\
& +\sum_{\alpha, \beta} \frac{1}{2 \Omega_{\alpha 0} \Omega_{\beta 0}} f_{i 0}^{\alpha} f_{j 0}^{\beta}\left[\frac{1}{\left(\Omega_{\alpha 0}+\Omega_{\beta 0}\right)^{2}} \dot{\mathcal{V}}_{\alpha \beta}(0) \sin \left[\left(\Omega_{\alpha 0}+\Omega_{\beta 0}\right) t\right]\right. \\
& +\frac{1}{\left(\Omega_{\alpha 0}+\Omega_{\beta 0}\right)^{3}}\left(\ddot{\mathcal{V}}_{\alpha \beta}(t)-\ddot{\mathcal{V}}_{\alpha \beta}(0) \cos \left[\left(\Omega_{\alpha 0}+\Omega_{\beta 0}\right) t\right]\right) \\
& \left.\left.+\frac{1}{\left(\Omega_{\alpha 0}+\Omega_{\beta 0}\right)^{3}} \int d t^{\prime} \dddot{\mathcal{V}}_{\alpha \beta}\left(t^{\prime}\right) \cos \left[\left(\Omega_{\alpha 0}+\Omega_{\beta 0}\right)\left(t-t^{\prime}\right)\right]\right]\right\} .
\end{aligned}
$$

The cancellation of the terms which would produce a singularity at $t=0$ then requires

$$
\begin{aligned}
\operatorname{Im} \rho^{\alpha \beta} & =\frac{1}{2 \Omega_{\alpha 0} \Omega_{\beta 0}} \frac{1}{\left(\Omega_{\alpha 0}+\Omega_{\beta 0}\right)^{2}} \dot{\mathcal{V}}_{\alpha \beta}(0), \\
\operatorname{Re} \rho^{\alpha \beta} & =\frac{1}{2 \Omega_{\alpha 0} \Omega_{\beta 0}} \frac{1}{\left(\Omega_{\alpha 0}+\Omega_{\beta 0}\right)^{3}} \ddot{\mathcal{V}}_{\alpha \beta}(0) .
\end{aligned}
$$


If the fields were independent $\mathcal{V}_{\alpha \beta}$ would be diagonal and we would obtain (omitting the indices of the diagonal elements)

$$
\frac{\operatorname{Im} \rho}{\operatorname{Re} \rho}=2 \Omega_{0} \frac{\dot{\mathcal{V}}(0)}{\ddot{\mathcal{V}}(0)}
$$

and

$$
|\rho|=\frac{1}{8 \Omega_{0}^{3}} \sqrt{\dot{\mathcal{V}}(0)^{2}+\frac{\ddot{\mathcal{V}}(0)^{2}}{4 \Omega_{0}^{2}}}
$$

for the separate Bogoliubov transformations of the two fields. This agrees in the approximation of large momenta with the results for the one-field case, Eqs. (51) and (52) of Ref. [2]. There it was possible to remove the contributions proportional to $\dot{\mathcal{V}}(0)$ and $\ddot{\mathcal{V}}(0)$ for all momenta. Here these terms are cancelled at large momenta only.

With Eqs. (3.9) and (3.10) we have obtained a solution to our problem of initial singularities. We have to stress that there is an infinite manifold of such solutions, differing, e.g., by a different choice of initial occupation numbers at finite momenta. They all have to share the same large momentum behavior, however.

Once we have $\rho$ we now must determine $M$, using (3.6), without any approximation, because otherwise our transformation would not be canonical. Though $\rho$ appears nonlinearly, Eq.(3.6) is simply a system of four linear equations for the matrix elements of $M$. Finally, the fluctuation integral (3.7) has to be computed using the exact numerical solutions $f_{i}^{\alpha}(t)$ in all four terms of the integrand.

For the numerical computations it is preferable to implement the Bogoliubov transformation in a different way, by redefining the mode functions. For this purpose we introduce

$$
\tilde{f}_{i}^{\gamma}(k, t)=\sum_{\alpha} \frac{\sqrt{2 \Omega_{\gamma 0}}}{\sqrt{2 \Omega_{\alpha 0}}}\left[f_{i}^{\alpha}(k, t) N_{\alpha \gamma}+f_{i}^{\alpha *}(k, t) \rho^{\alpha \kappa *} N_{\kappa \gamma}\right],
$$

where the matrix $N$ satisfies $N \times N=M 3$. It can be determined using the eigenvalues and eigenvectors of $M$. One easily verifies that

$$
\tilde{\mathcal{F}}_{i j}(t)=\sum_{\alpha} \int \frac{d^{3} k}{(2 \pi)^{3} 2 \Omega_{\gamma 0}} \tilde{f}_{i}^{\gamma}(k, t) \tilde{f}_{j}^{\gamma *}(k, t)
$$

\footnotetext{
${ }^{3} N$ is not uniquely determined, all we need is one particular matrix that satisfies this relation. As $M$ is Hermitian, so is $N$.
} 
is identical to the previous definition, Eq. (3.7). As $f_{i}^{\alpha}$ and $f_{i}^{\alpha *}$ are solutions of the same equation of motion, Eq. (2.13), so is $\tilde{f}_{i}^{\gamma}$. It is sufficient, therefore, to determine $\tilde{f}_{i}^{\gamma}$ as a solution to this equation with the initial conditions implied by Eqs. (3.13), (2.14) and (2.15).

Having presented the technical procedure we would like to add some comments concerning the interpretation. In order to do so we need to discuss the problem of initial states in a more general way.

The adiabatic vacuum has often been used as an initial state for preheating simulations, maybe on the grounds that after inflation one ends up in a temperature zero state, i.e. an "empty" vacuum. Indeed if this is the case, and if the evolution of the classical fields is very slow ("adiabatic"), this can be considered to be a reasonable guess for an initial state. Another choice that may be reasonable, e.g. after thermalization and in a period of adiabatic evolution, is a thermal initial state. This is of course not a pure state. For a thermal state the fluctuation integral would be replaced by

$$
\mathcal{F}_{i j}(t)=\sum_{\alpha} \int \frac{d^{3} k}{(2 \pi)^{3} 2 \Omega_{\alpha 0}(k)}\left(2 N_{\alpha}(k)+1\right) f_{i}^{\alpha}(k, t) f_{j}^{\alpha *}(k, t),
$$

with

$$
N_{\alpha}(k)=\left[\exp \left(\Omega_{\alpha}(k) / T\right)-1\right]^{-1} .
$$

Here we have used the fact that the mass matrix and therefore the fluctuation Hamiltonian is diagonal in the basis $f_{i 0}^{\alpha}$ at $t=0$.

If one takes into account the real evolution of the system before $t=0$ then neither the adiabatic vacuum nor a thermal initial state will be appropriate. If the system has started, before $t=0$, in a pure quantum state, it cannot have ended up, at $t=0$, in a thermal state or in any other state described simply by particle numbers $N_{\alpha}(k)$. The quantum system can be interpreted as a system of independent free particle only after "decoherence", a concept that has been addressed in the present context in Refs. [37, 38]. But even if the system has started, at an earlier time, with a mixed state, the interaction with the background field will have created a coherence in the different components of such a state at $t=0$, and a representation in the form (3.15) will not be possible.

So, if one takes into account the evolution of the system prior to $t=0$ then one would have to know the entire prehistory or at least the prehistory of a long period in order to describe the state at $t=0$ with its full quantum coherence. This is of course not possible unless one knows how to start the 
system at an earlier time, facing then the same problem. The best one can hope for is that after some time the system will not remember much of its initial state. This is presumably the case if the background fields produce large quantum fluctuations at later times.

The purpose of the Bogoliubov transformation is different. If we know the evolution of the background fields near $t=0$ (and by continuity this means also shortly before $t=0$ ), to the extent that we know $\dot{\mathcal{V}}(0)$ and $\ddot{\mathcal{V}}(0)$, or, equivalently, $\dot{\phi}(0)$ and $\ddot{\phi}(0)$, then we have a limited information on the initial state. Constant background fields at $t<0$ and the adiabatic vacuum state as initial state would produce a singularity of the first two derivatives of the Green's function. The Bogoliubov transformation removes this singularity, or, more precisely, it reduces it to higher orders in the derivatives. In this way it takes a minimal account of the fact that the system is not static before $t=0$. As we have displayed above the transformation may be considered as a modification of the state or of the mode functions. The new state should not be considered as a vacuum state. The adiabatic vacuum state remains the lowest energy state for a given set of background fields. The analysis of the high momentum behavior of the fluctuation integral simply shows that the system will never arrive at this state if the background fields keep changing with time. In reality, of course, we would rather expect the quantum state of the fluctuations to be an excited one, particularly at low momentum. Our simple requirement of continuity for the Green's function does not give us any information on this excited state, except at high momenta.

The state generated by the Bogoliubov transformation applied to the adiabatic vacuum is a pure state. Therefore, it cannot be described by a mixed state with suitable particle numbers $N_{\alpha}(k)$. If for some physical motivation we want to start with a thermal state or some other state specified by particle numbers we have to combine two different concepts: a mixed state made up of different excited Fock-space states and a pure state that ensures the continuity of the Green's function. For a thermal state the particle numbers decrease exponentially as $k \rightarrow \infty$; then the discontinuity of the Green's function solely arises from the vacuum contribution. Its Bogoliubov transformation is well-defined and compulsory at high momenta only. There are then, among many others, two pragmatic ways of defining a thermal initial state: (i) One defines the thermal state using for all momenta the modified mode functions $\tilde{f}_{i}^{\alpha}(k, t)$. This is not a quite a thermal state, though, as the modified mode functions are not eigenfunctions of $i \partial / \partial t$. (ii) One uses the integrand of Eq. (3.15) with the original mode functions $f_{i}^{\alpha}(k, t)$ for low 
momenta only, and the integrand of Eq. (3.14) at high momenta.

\section{The energy-momentum tensor}

The energy-momentum tensor for the fluctuations $t_{\mu \nu}=\left(T^{\text {fluc }}\right)_{\mu \nu}$ in a homogeneous background field is diagonal and has identical space-space components. It may be specified by the two independent expectation values

$$
\begin{aligned}
t_{00}=\epsilon= & \frac{1}{2}<\dot{\psi}_{i}(\mathbf{x}, t) \dot{\psi}_{i}(\mathbf{x}, t) \\
& +\vec{\nabla} \psi_{i}(\mathbf{x}, t) \vec{\nabla} \psi_{i}(\mathbf{x}, t)+\mathcal{M}_{i j}^{2}(t) \psi_{i}(\mathbf{x}, t) \psi_{j}(\mathbf{x}, t)>
\end{aligned}
$$

and

$$
\begin{aligned}
t_{\mu}^{\mu}=\epsilon-3 p= & <-\dot{\psi}_{i}(\mathbf{x}, t) \dot{\psi}_{i}(\mathbf{x}, t) \\
& +\vec{\nabla} \psi_{i}(\mathbf{x}, t) \vec{\nabla} \psi_{i}(\mathbf{x}, t)+2 \mathcal{M}_{i j}^{2}(t) \psi_{i}(\mathbf{x}, t) \psi_{j}(\mathbf{x}, t)>.
\end{aligned}
$$

$\epsilon$ is the energy density and $p$ the pressure. $t_{00}$ and $t_{\mu}^{\mu}$ can be evaluated in the adiabatic vacuum and in the Bogoliubov-transformed vacuum in the same way as the fluctuation integrals. We just present the expectation values in the Bogoliubov-transformed vacuum, the one in the adiabatic vacuum is obtained by substituting $\rho \rightarrow 0$ and $M \rightarrow I$. For $\tilde{t}_{00}$ we obtain

$$
\begin{aligned}
& \tilde{t}_{00}=\int \frac{d^{3} k}{(2 \pi)^{3}} \sum_{\alpha, \beta} \frac{1}{4 \Omega_{\alpha 0}}\left\{\operatorname{Re}\left[\rho^{\beta \kappa} M_{\alpha \kappa}\left(\dot{f}_{i}^{\alpha} \dot{f}_{i}^{\beta}+k^{2} f_{i}^{\alpha} f_{i}^{\beta}+\mathcal{M}_{i j}^{2} f_{i}^{\alpha} f_{j}^{\beta}\right)\right]\right. \\
& \left.+\left[M_{\alpha \beta}+M_{\kappa \lambda} \rho^{\beta \kappa *} \rho^{\alpha \lambda}\right]\left(\dot{f}_{i}^{\alpha} \dot{f}_{i}^{\beta *}+k^{2} f_{i}^{\alpha} f_{i}^{\beta *}+\mathcal{M}_{i j}^{2} f_{i}^{\alpha} f_{j}^{\beta *}\right)\right\}
\end{aligned}
$$

For the trace we find

$$
\begin{aligned}
& \tilde{t}_{\mu}^{\mu}=\int \frac{d^{3} k}{(2 \pi)^{3}} \sum_{\alpha, \beta} \frac{1}{4 \Omega_{\alpha 0}}\left\{\operatorname{Re}\left[\rho^{\beta \kappa} M_{\alpha \kappa}\left(-\dot{f}_{i}^{\alpha} \dot{f}_{i}^{\beta}+k^{2} f_{i}^{\alpha} f_{i}^{\beta}+2 \mathcal{M}_{i j}^{2} f_{i}^{\alpha} f_{j}^{\beta}\right)\right]\right. \\
& \left.+\left[M_{\alpha \beta}+M_{\kappa \lambda} \rho^{\beta \kappa *} \rho^{\alpha \lambda}\right]\left(-\dot{f}_{i}^{\alpha} \dot{f}_{i}^{\beta *}+k^{2} f_{i}^{\alpha} f_{i}^{\beta *}+2 \mathcal{M}_{i j}^{2} f_{i}^{\alpha} f_{j}^{\beta *}\right)\right\} .
\end{aligned}
$$

Both expressions can alternatively be rewritten in terms of the modified mode functions of Eq. (3.13). 
Using the equation of motion for the fluctuations we can write

$$
\begin{aligned}
-\dot{f}_{i}^{\alpha} \dot{f}_{i}^{\beta *}+k^{2} f_{i}^{\alpha} f_{i}^{\beta *}+\mathcal{M}_{i j}^{2} f_{i}^{\alpha} f_{j}^{\beta *} & =-\frac{1}{2} \frac{d^{2}}{d t^{2}} f_{i}^{\alpha} f_{j}^{\beta *}, \\
-\dot{f}_{i}^{\alpha} \dot{f}_{i}^{\beta}+k^{2} f_{i}^{\alpha} f_{i}^{\beta}+\mathcal{M}_{i j}^{2} f_{i}^{\alpha} f_{j}^{\beta} & =-\frac{1}{2} \frac{d^{2}}{d t^{2}} f_{i}^{\alpha} f_{j}^{\beta} .
\end{aligned}
$$

Therefore the trace can be expressed in terms of the fluctuation integrals (3.7) as

$$
\tilde{t}_{\mu}^{\mu}=-\frac{1}{2} \frac{d^{2}}{d t^{2}} \tilde{\mathcal{F}}_{i i}+\mathcal{M}_{i j} \tilde{\mathcal{F}}_{i j} .
$$

Both the energy density and the trace contain second space and time derivatives of the two-point function and this can transform the mild singularities found in the fluctuation integrals $\mathcal{F}_{i j}$ into infinities at $t=0$. In Ref. [2] it was found that the energy density remains finite even in the adiabatic vacuum. As the second derivatives only appear in the kinetic terms which are diagonal this analysis remains valid for the coupled-channel case. However, the trace of the energy-momentum tensor $t_{\mu}^{\mu}$ in the adiabatic vacuum contains the second time derivative of the fluctuation integrals $\mathcal{F}_{i i}$ and this behaves as $d^{2}(t \ln t) / d t^{2}=1 / t$ as $t \searrow 0$. In the transformed fluctuation integrals $\tilde{\mathcal{F}}_{i j}$ we have removed the dangerous terms, and so $\tilde{t}_{\mu}^{\mu}$ has a finite value at $t=0$.

When the field is coupled to gravity [39] the expressions (4.1) and (4.2) receive some further contributions that we do not want to discuss here in detail. They can be written in terms of the fluctuation integrals and their first derivatives. The fluctuation integrals themselves are not infinite at $t=0$. The most singular of the additional terms are proportional to $\left(\xi_{i}-1 / 6\right) H d \mathcal{F}_{i i} / d t$ and appear both in $t_{00}$ and $t_{\mu}^{\mu}$. They behave as $\ln t$ as

$t \searrow 0$; in the Bogoliubov-transformed initial state $\mathcal{F}_{i i}$ is replaced by $\tilde{\mathcal{F}}_{i i}$ and then the energy-momentum tensor remains finite at $t=0$.

\section{The adiabatic particle number}

The adiabatic particle number is obtained by representing the fluctuation field at time $t$ in terms of the adiabatic Fock space at time $t$. The fluctuation field is given by Eq. (2.17). The adiabatic Fock space at time $t$ is defined in terms of particle excitations which are eigenstates of the mass matrix $\mathcal{M}_{i j}^{2}(t)$. We define the eigenvectors of the mass matrix by

$$
\mathcal{M}_{i j}^{2}(t) f_{j t}^{\alpha}=m_{\alpha t}^{2} f_{i t}^{\alpha},
$$


we again choose them to be real and normalized via

$$
\sum_{i} f_{i t}^{\alpha} f_{i t}^{\beta}=\delta^{\alpha \beta}
$$

and define $\Omega_{\alpha t}=\sqrt{k^{2}+m_{\alpha t}^{2}}$. We further expand the fields with respect to the new basis as

$$
\begin{aligned}
& \psi_{i}(\mathbf{x}, t)=\sum_{\alpha} \int \frac{d^{3} k}{(2 \pi)^{3} 2 \Omega_{\alpha t}}\left[a_{\alpha t}(\mathbf{k}) f_{i t}^{\alpha}+a_{\alpha t}^{\dagger}(-\mathbf{k}) f_{i t}^{\alpha}\right] e^{i \mathbf{k x}}, \\
& \dot{\psi}_{i}(\mathbf{x}, t)=-i \sum_{\alpha} \int \frac{d^{3} k}{(2 \pi)^{3} 2}\left[a_{\alpha t}(\mathbf{k}) f_{i t}^{\alpha}-a_{\alpha t}^{\dagger}(-\mathbf{k}) f_{i t}^{\alpha}\right] e^{i \mathbf{k x}},
\end{aligned}
$$

where we have chosen the initial conditions for the modes $f_{i t}^{\alpha}(k, t)$ in analogy to Eqs. (2.14) and (2.15). Using the field expansion the new annihilation operators $a_{\alpha t}(\mathbf{k})$ can be expressed as

$$
a_{\alpha t}(\mathbf{k})=\int d^{3} x e^{-i \mathbf{k x}}\left[\Omega_{\alpha t} \psi_{i}(\mathbf{x}, t)+i \dot{\psi}_{i}(\mathbf{x}, t)\right] f_{i t}^{\alpha} .
$$

They annihilate the adiabatic vacuum defined at time $t$. The relation to the original operators $a_{\alpha}(\mathbf{k})$ is obtained by inserting the field expansion (2.17) into Eq. (5.5). We find

$$
a_{\alpha t}(\mathbf{k})=\sum_{\beta} \sqrt{\frac{\Omega_{\alpha t}}{\Omega_{\beta 0}}}\left[C^{\alpha \beta} a_{\beta}(\mathbf{k})-S^{\alpha \beta} a_{\beta}^{\dagger}(-\mathbf{k})\right],
$$

with

$$
\begin{aligned}
C^{\alpha \beta} & =\frac{1}{2 \sqrt{\Omega_{\alpha t} \Omega_{\beta 0}}}\left[\Omega_{\alpha t} f_{i}^{\beta}(k, t)+i \dot{f}_{i}^{\beta}(k, t)\right] f_{i t}^{\alpha}, \\
S^{\alpha \beta} & =\frac{-1}{2 \sqrt{\Omega_{\alpha t} \Omega_{\beta 0}}}\left[\Omega_{\alpha t} f_{i}^{\beta *}(k, t)+i \dot{f}_{i}^{\beta *}(k, t)\right] f_{i t}^{\alpha} .
\end{aligned}
$$

Using the relations $(\overline{B .8}),(\underline{B .9})$ and $(\bar{B} .10)$ of Appendix B it is straightforward to verify that this is a Bogoliubov transform, i.e., that Eqs. (A.23) and (A.26) are satisfied. In terms of the matrices $C$ and $S$ the adiabatic particle number density is given by 4 ,

$$
n_{\alpha}(k, t)=\frac{1}{2 V \Omega_{\alpha t}}<0\left|a_{\alpha t}^{\dagger}(\mathbf{k}) a_{\alpha t}(\mathbf{k})\right| 0>=\sum_{\gamma} S^{\alpha \gamma *} S^{\alpha \gamma} .
$$

\footnotetext{
${ }^{4}$ No summation over $\alpha$. For simplicity of presentation we assume the adiabatic vacuum as initial state.
} 
Inserting Eq. (5.8) we obtain

$$
n_{\alpha}(k, t)=\frac{1}{2 \Omega_{\alpha t}} \sum_{\gamma} \frac{1}{2 \Omega_{\gamma 0}}\left[\Omega_{\alpha t}^{2} f_{i}^{\gamma} f_{j}^{\gamma *}+\dot{f}_{i}^{\gamma} \dot{f}_{j}^{\gamma *}\right] f_{i t}^{\alpha} f_{j t}^{\alpha} .
$$

This has a simple interpretation: one decomposes the energy density with respect to the fluctuations $f_{i t}^{\alpha}$. Then $n_{\alpha}(k, t)$ is obtained by dividing the part corresponding to the fluctuations $f_{i t}^{\alpha}$ by the frequency $\Omega_{\alpha t}$ of these fluctuations. This result is analogous to the one-channel case.

While the definition (5.9) is suggestive we would like to add that this particle number does not imply a representation of the fluctuation integral in the form (3.15) in terms of the mode functions $f_{i t}^{\alpha}(k, t)$. Indeed, if one wants to use the representation (5.3) for calculating the fluctuation integral one gets nontrivial contributions from the operators $a_{\alpha t}(\mathbf{k}) a_{\alpha t}\left(\mathbf{k}^{\prime}\right), a_{\alpha t}^{\dagger}(\mathbf{k}) a_{\alpha t}^{\dagger}\left(\mathbf{k}^{\prime}\right)$ and

$a_{\alpha t}^{\dagger}(\mathbf{k}) a_{\alpha t}^{\dagger}\left(\mathbf{k}^{\prime}\right)$ as well, see Eqs. (A.38), (A.39) and (A.40). These contributions to the fluctuation integral are negligible for large particle numbers only, i.e., if the matrix elements $S^{\alpha \gamma}$ are much larger than the $C^{\alpha \gamma}$.

\section{Summary}

We have addressed here two topics of the nonequilibrium dynamics of coupled fields in a one-loop approximation to quantum backreaction: the problem of the initial time singularity in the energy-momentum tensor and the definition of the adiabatic particle number for a system of coupled scalar fields. Along with these topics we have considered Bogoliubov transformations and some aspects of the canonical formalism for such coupled systems.

Our main interest, as evident from the title, were the initial time singularities. We have been able to define a Bogoliubov transformation of the initial state that removes the initial time singularities in such a way that the energy-momentum tensor is finite in the limit $t \searrow 0$. This is important if one considers the evolution of such a system of fields coupled to gravity. Clearly, this Bogoliubov transformation is constrained only at large momenta. So modifications that are subleading at high momenta are still acceptable. We had to include a discussion of the canonical formalism for a coupled-channel system, as some of the results were needed in the construction of the initial time singularities: we had to ensure that the fluctuation integrals are real and symmetric in the indices, as they should be on account of their definition. 
Both the discussion of the canonical formalism and of Bogoliubov transformations for coupled-channel systems are at the same time the basis for defining the adiabatic particle number density. So we have derived an expression for this density in terms of the coupled system mode functions. It is analogous to the definition in the single-channel case and has a simple intuitive interpretation. Another formulation for the adiabatic particle number, based in an eikonal formalism and the evolution of Bogoliubov coefficients, has been presented recently [24]. As both formalisms are canonical, the results should be equivalent, though it may be difficult to verify this analytically.

\section{Acknowledgments}

One of us (N.K.) thanks the Humboldt Foundation for financial support, and the Deutsche Elektronensynchrotron DESY, Hamburg, for hospitality.

\section{A The Bogoliubov transformation for a cou- pled system}

We first recall some basic relations for the case of a single quantum field, see, e.g., Ref. [40]. The Bogoliubov transformation rotates creation into annihilation operators and vice versa, such as to preserve the canonical commutation relations

$$
\begin{aligned}
{\left[a(\mathbf{k}), a\left(\mathbf{k}^{\prime}\right)\right] } & =0 \\
{\left[a(\mathbf{k}), a^{\dagger}\left(\mathbf{k}^{\prime}\right)\right] } & =(2 \pi)^{3} 2 \omega \delta^{3}\left(\mathbf{k}-\mathbf{k}^{\prime}\right) \\
{\left[a^{\dagger}(\mathbf{k}), a^{\dagger}\left(\mathbf{k}^{\prime}\right)\right] } & =0 .
\end{aligned}
$$

Furthermore the transformation has to be chosen in such a way that the vacuum retains its total momentum zero and remains isotropic. The most general form of such a transformation is then induced by the operator

$$
Q=\frac{1}{2} \int \frac{d^{3} k}{(2 \pi)^{3} 2 \omega}\left[q(k) a^{\dagger}(\mathbf{k}) a^{\dagger}(-\mathbf{k})-q^{*}(k) a(\mathbf{k}) a(-\mathbf{k})\right],
$$

via

$$
\tilde{a}(\mathbf{k})=\exp (Q) a(\mathbf{k}) \exp \left(Q^{\dagger}\right)=\exp (Q) a(\mathbf{k}) \exp (-Q)
$$


and

$$
|\tilde{0}>=\exp (Q)| 0>.
$$

Here $q(k)$ is a general complex function of $k=|\mathbf{k}|$. We have

$$
\begin{aligned}
{[a(\mathbf{k}), Q] } & =q(k) a^{\dagger}(-\mathbf{k}), \\
{\left[a^{\dagger}(-\mathbf{k}), Q\right] } & =q^{*}(k) a(\mathbf{k}) .
\end{aligned}
$$

We have in general

$$
a(\mathbf{k}) \exp (-Q)=\exp (-Q) \sum_{n=1}^{\infty} \frac{(-1)^{n}}{n !}\left[[[[[a(\mathbf{k}), Q], Q] \ldots], Q]_{n},\right.
$$

where the $n$-th term in the sum contains $n$ commutators. The even commutators $(n=2 l)$ yield

$$
\left[[[[[a(\mathbf{k}), Q], Q] \ldots], Q]_{2 l}=|q(k)|^{2 l} a(\mathbf{k}),\right.
$$

the odd ones $(n=2 l+1)$ yield

$$
[[[[a(\mathbf{k}), Q], Q] \ldots], Q]_{2 l+1}=|q(k)|^{2 l} q(k) a^{\dagger}(-\mathbf{k}) .
$$

Writing $q(k)=\gamma(k) e^{i \delta(k)}$ with real constants $\gamma$ and $\delta$ we find

$a(\mathbf{k}) \exp (-Q)=\exp (-Q)\left[\cosh (\gamma) a(\mathbf{k})-\sinh (\gamma) e^{i \delta} a^{\dagger}(-\mathbf{k})\right]=\exp (-Q) \tilde{a}(\mathbf{k})$.

With these preliminaries the generalization is straightforward. We have two sets of creation and annihilation operators $a_{\alpha}^{\dagger}(\mathbf{k})$ and $a_{\alpha}(\mathbf{k})$, where $\alpha=$ 1,2 labels the two independent solutions $f_{i}^{\alpha}(k)$. We have the field expansion

$$
\psi_{i}(\mathbf{x}, t)=\int \frac{d^{3} k}{(2 \pi)^{3}} \sum_{\alpha} \frac{1}{2 \Omega_{\alpha 0}}\left\{a_{\alpha}(\mathbf{k}) f_{i}^{\alpha}(k, t) e^{i \mathbf{k x}}+a_{\alpha}^{\dagger}(-\mathbf{k}) f_{i}^{\alpha *}(k, t) e^{-i \mathbf{k x}}\right\}
$$

and the commutation relations

$$
\left[a_{\alpha}(\mathbf{k}), a_{\beta}^{\dagger}\left(\mathbf{k}^{\prime}\right)\right]=(2 \pi)^{3} 2 \Omega_{\alpha 0} \delta_{\alpha \beta} \delta^{3}\left(\mathbf{k}-\mathbf{k}^{\prime}\right) .
$$

The operator $Q$ now takes the form

$$
Q=\frac{1}{2} \int \frac{d^{3} k}{(2 \pi)^{3}} \sum_{\alpha, \beta} \frac{1}{2 \sqrt{\Omega_{\alpha 0} \Omega_{\beta 0}}}\left[q^{\alpha \beta}(k) a_{\alpha}^{\dagger}(\mathbf{k}) a_{\beta}^{\dagger}(-\mathbf{k})-q^{\alpha \beta *}(k) a_{\alpha}(\mathbf{k}) a_{\beta}(-\mathbf{k})\right] .
$$


The normalization convention introduced by writing $\sqrt{\Omega_{\alpha 0} \Omega_{\beta 0}}$ has the advantage of keeping the functions $q^{\alpha \beta}(k)$ symmetric in the indices. Indeed this symmetry is the only restriction on these functions; as they are complex we have six free parameters, which are functions of $k$. The symmetry arises from the fact that the products $a^{\alpha}(\mathbf{k}) a^{\beta}(-\mathbf{k})$ and $a^{\beta}(\mathbf{k}) a^{\alpha}(-\mathbf{k})$ are equivalent. On the one hand the operators commute, and on the other hand the arguments $\mathbf{k}$ and $\mathbf{- k}$ may be exchanged as the integration is symmetric in the sign of $\mathbf{k}$ and the functions $q^{\alpha \beta}$ only depend on $|\mathbf{k}|$. An asymmetric part of these functions would simply be summed and integrated away. We again have $Q^{\dagger}=-Q$ and the transformation matrix $\exp (Q)$ is unitary.

What does not work here, at least not in a general parameterization $q^{\alpha \beta}$, is the explicit evaluation of the transformation of the annihilation and creation operators. The matrix $q^{\alpha \gamma} q^{\gamma \beta *}$ which appears after every second step in the evaluation of the multiple commutators, is given by

$$
q^{\alpha \gamma} q^{\gamma \beta *}=\left(\begin{array}{cc}
\left|q^{11}\right|^{2}+\left|q^{12}\right|^{2} & q^{11} q^{12 *}+q^{12} q^{22 *} \\
q^{11 *} q^{12}+q^{12 *} q^{22} & \left|q^{12}\right|^{2}+\left|q^{22}\right|^{2}
\end{array}\right)^{\alpha \beta}
$$

It is Hermitian, in analogy to the reality of $|q|^{2}$ in the single-channel case. It is diagonal in two cases: (i) $q^{12}=0$ and (ii) $q^{11}=q^{22}=0$. It is instructive to evaluate the transformation of $a_{\alpha}(\mathbf{k})$ in the two cases. In the first case we find

$$
\begin{aligned}
& \tilde{a}_{1}(\mathbf{k})=\cosh \left(\left|q^{11}\right|\right) a_{1}(\mathbf{k})-\sinh \left(\left|q^{11}\right|\right) e^{i \arg \left(q^{11}\right)} a_{1}^{\dagger}(-\mathbf{k}) \\
& \tilde{a}_{2}(\mathbf{k})=\cosh \left(\left|q^{22}\right|\right) a_{2}(\mathbf{k})-\sinh \left(\left|q^{22}\right|\right) e^{i \arg \left(q^{22}\right)} a_{2}^{\dagger}(-\mathbf{k})
\end{aligned}
$$

i.e., a simple Bogoliubov transformation for each channel. In the second case we have

$$
\begin{aligned}
& \tilde{a}_{1}(\mathbf{k})=\cosh \left(\left|q^{12}\right|\right) a_{1}(\mathbf{k})-\sinh \left(\left|q^{12}\right|\right) e^{i \arg \left(q^{12}\right)} \sqrt{\frac{\Omega_{10}}{\Omega_{20}}} a_{2}^{\dagger}(-\mathbf{k}), \\
& \tilde{a}_{2}(\mathbf{k})=\cosh \left(\left|q^{12}\right|\right) a_{2}(\mathbf{k})-\sinh \left(\left|q^{12}\right|\right) e^{i \arg \left(q^{12}\right)} \sqrt{\frac{\Omega_{20}}{\Omega_{10}}} a_{1}^{\dagger}(-\mathbf{k})
\end{aligned}
$$

i.e., an annihilation operator in channel 1 is mixed with a creation operator in channel 2 .

In the general case the matrix $q^{\alpha \gamma} q^{\gamma \beta *}$ is not diagonal. Still we can sum up the series formally, as a series of matrix products. As the exponential 
series converges well this can be done even numerically. We write

$$
\tilde{a}_{\alpha}(\mathbf{k})=\sum_{\beta} \sqrt{\frac{\Omega_{\alpha 0}}{\Omega_{\beta 0}}}\left[C^{\alpha \beta} a_{\beta}(\mathbf{k})-S^{\alpha \beta} a_{\beta}^{\dagger}(-\mathbf{k})\right] .
$$

In terms of the matrix $q^{\alpha \beta}$ we then have

$$
C^{\alpha \beta}=\sum_{n} \frac{1}{(2 n) !}\left[(q q *)^{n}\right]^{\alpha \beta}
$$

where $q q *$ is the matrix product $q^{\alpha \gamma} q^{\gamma \beta *}$ and the power series is a series of powers of this matrix. Further, we have

$$
S^{\alpha \beta}=\sum_{n} \frac{1}{(2 n+1) !}\left[(q q *)^{n}\right]^{\alpha \gamma} q^{\gamma \beta} .
$$

Instead of writing these matrices as power series in $q^{\alpha \beta}$ we can ask for the conditions on $C$ and $S$ that follow from the requirement that the commutation rules should be conserved. From

$$
\left[a_{\alpha}(\mathbf{k}), a_{\beta}\left(\mathbf{k}^{\prime}\right)\right]=0
$$

one finds

$$
\sum_{\gamma}\left(C^{\alpha \gamma} S^{\beta \gamma}-S^{\alpha \gamma} C^{\beta \gamma}\right)=0
$$

or

$$
C S^{\top}=S C^{\top}=\left(C S^{\top}\right)^{\top}
$$

Multiplying from the left with $C^{-1}$ and from the right with $\left(C^{\mathbf{\top}}\right)^{-1}$ one finds

$$
C^{-1} S=\left(C^{-1} S\right)^{\top}
$$

i.e., this a symmetric matrix.

Considering the nonvanishing commutator we find

$$
\sum_{\gamma}\left(C^{\alpha \gamma} C^{\beta \gamma *}-S^{\alpha \gamma} S^{\beta \gamma *}\right)=\delta_{\alpha \beta},
$$

or, in matrix form,

$$
C C^{\dagger}-S S^{\dagger}=I
$$


the obvious generalization of

$$
\cosh ^{2}(\gamma)-\sinh ^{2}(\gamma)=1
$$

Instead of having to deal with two matrices it may be more convenient to deal with just one: the condition that the operators $\tilde{a}_{\alpha}(\mathbf{k})$ annihilate the vacuum $\mid \tilde{0}>$ reads

$$
\tilde{a}_{\gamma}(\mathbf{k})\left|\tilde{0}>=\sqrt{\frac{\Omega_{\gamma 0}}{\Omega_{\alpha 0}}} C^{\gamma \alpha}\left[a_{\alpha}(\mathbf{k})-\sqrt{\frac{\Omega_{\alpha 0}}{\Omega_{\beta 0}}} \rho^{\alpha \beta} a_{\beta}^{\dagger}(-\mathbf{k})\right]\right| \tilde{0}>=0,
$$

where we have defined the matrix

$$
\rho=C^{-1} S
$$

From Eq. A.25 we see that $\rho$ is a symmetric matrix. Indeed we had found previously that we have six free parameters for the most general transformation.

To begin with we compute the expectation value of $a_{\alpha}(\mathbf{k}) a_{\alpha}^{\dagger}\left(\mathbf{k}^{\prime}\right)$ in the new vacuum. As vacua are homogeneous and isotropic we can write

$$
<\tilde{0}\left|a_{\alpha}(\mathbf{k}) a_{\beta}^{\dagger}\left(\mathbf{k}^{\prime}\right)\right| \tilde{0}>=(2 \pi)^{3} \delta^{3}\left(\mathbf{k}-\mathbf{k}^{\prime}\right) 2 \sqrt{\Omega_{\alpha 0} \Omega_{\beta 0}} M_{\alpha \beta}(k) .
$$

This definition implies that $M$ is a Hermitian matrix. Using the commutation relations and (A.29) we have

$$
\begin{aligned}
& <\tilde{0}\left|a_{\alpha}(\mathbf{k}) a_{\beta}^{\dagger}\left(\mathbf{k}^{\prime}\right)\right| \tilde{0}> \\
= & (2 \pi)^{3} 2 \Omega_{\alpha 0} \delta^{3}\left(\mathbf{k}-\mathbf{k}^{\prime}\right) \delta_{\alpha \beta}+<\tilde{0}\left|a_{\beta}^{\dagger}\left(\mathbf{k}^{\prime}\right) a_{\alpha}(\mathbf{k})\right| \tilde{0}> \\
= & (2 \pi)^{3} 2 \Omega_{\alpha 0} \delta^{3}\left(\mathbf{k}-\mathbf{k}^{\prime}\right) \delta_{\alpha \beta}+\sqrt{\frac{\Omega_{\alpha 0} \Omega_{\beta 0}}{\Omega_{\kappa 0} \Omega_{\lambda 0}}} \rho^{\alpha \kappa} \rho^{\beta \lambda *}<\tilde{0}\left|a_{\lambda}\left(-\mathbf{k}^{\prime}\right) a_{\kappa}^{\dagger}(-\mathbf{k})\right| \tilde{0}>.
\end{aligned}
$$

In terms of the matrix $M$ we find

$$
M_{\alpha \beta} \sqrt{\Omega_{\alpha 0} \Omega_{\beta 0}}=\delta_{\alpha \beta} \Omega_{\alpha 0}+\sqrt{\Omega_{\alpha 0} \Omega_{\beta 0}} \rho^{\alpha \kappa} \rho^{\beta \lambda *} M_{\lambda \kappa},
$$

or

$$
M-\rho M^{\top} \rho^{\dagger}=I .
$$

This can be solved explicitly for $M$. Using the symmetry of $\rho$ it easy to verify, e.g., using the series expansion in $\rho \rho^{\dagger}$, that

$$
M=\left(I-\rho \rho^{\dagger}\right)^{-1} .
$$


Furthermore, using $S=C \rho$ it is easy to see, using Eq. (A.27), that

$$
C C^{\dagger}=\left(I-\rho \rho^{\dagger}\right)^{-1}=M .
$$

This implies that knowing $\rho$ the matrix $C$ is not determined uniquely. If one uses a basis in which $\rho$ is diagonal, $C C^{\dagger}$ is diagonal as well, and we have two free phases in the matrix $C$. A further useful identity is

$$
M \rho=\rho M^{\top} .
$$

It can easily be verified using again the expansion of $M$ interms of $\rho \rho^{\dagger}$.

We next evaluate the expectation values of the other products:

$$
\begin{aligned}
&<\tilde{0}\left|a_{\alpha}(\mathbf{k}) a_{\beta}\left(\mathbf{k}^{\prime}\right)\right| \tilde{0}>=\sqrt{\frac{\Omega_{\beta 0}}{\Omega_{\kappa 0}}} \rho^{\beta \kappa}<\tilde{0}\left|a_{\alpha}(\mathbf{k}) a_{\kappa}^{\dagger}\left(-\mathbf{k}^{\prime}\right)\right| \tilde{0}> \\
&=(2 \pi)^{3} \delta^{3}\left(\mathbf{k}+\mathbf{k}^{\prime}\right) 2 \sqrt{\Omega_{\alpha 0} \Omega_{\beta 0}} \rho^{\beta \kappa} M_{\alpha \kappa}(k), \\
&=(2 \pi)^{3} \delta^{3}\left(\mathbf{k}+\mathbf{k}^{\prime}\right) 2 \sqrt{\Omega_{\alpha 0} \Omega_{\beta 0}} \rho^{\alpha \kappa *} M_{\kappa \beta}(k), \\
&<\tilde{0}\left|a_{\alpha}^{\dagger}(\mathbf{k}) a_{\beta}^{\dagger}\left(\mathbf{k}^{\prime}\right)\right| \tilde{0}>=\sqrt{\frac{\Omega_{\alpha 0}}{\Omega_{k 0}}} \rho^{\alpha \kappa *}<\tilde{0}\left|a_{\kappa}(-\mathbf{k}) a_{\beta}^{\dagger}\left(\mathbf{k}^{\prime}\right)\right| \tilde{0}> \\
&=(2 \pi)^{3} \delta^{3}\left(\mathbf{k}-a_{\alpha}^{\dagger}(\mathbf{k}) a_{\beta}\left(\mathbf{k}^{\prime}\right)\left|\tilde{0}>=\sqrt{\frac{\Omega_{\alpha 0} \Omega_{\beta 0}}{\Omega_{\lambda 0} \Omega_{\kappa 0}}} \rho^{\alpha \kappa *} \rho^{\beta \lambda}<\tilde{0}\right| a_{\kappa}(-\mathbf{k}) a_{\lambda}^{\dagger}\left(-\mathbf{k}^{\prime}\right) \mid \tilde{0}>\right.
\end{aligned}
$$

In terms of the matrices $\rho$ and $M$ the fluctuation integral, evaluated in the Bogoliubov-transformed vacuum takes the form

$$
\begin{aligned}
& \tilde{\mathcal{F}}_{i j}(t)=<\tilde{0}\left|\psi_{i}(\mathbf{x}, t) \psi_{j}(\mathbf{x}, t)\right| \tilde{0}> \\
= & \int \frac{d^{3} k}{(2 \pi)^{3}} \sum_{\alpha, \beta} \frac{1}{2 \sqrt{\Omega_{\alpha 0} \Omega_{\beta 0}}}\left[f_{i}^{\alpha}(t) f_{j}^{\beta}(t) \rho^{\beta \kappa} M_{\alpha \kappa}\right. \\
& +f_{i}^{\alpha}(t) f_{j}^{\beta *}(t) M_{\alpha \beta} \\
& +f_{i}^{\alpha *}(t) f_{j}^{\beta}(t) \rho^{\alpha \kappa *} \rho^{\beta \lambda} M_{\kappa \lambda} \\
& \left.+f_{i}^{\alpha *}(t) f_{j}^{\beta *}(t) \rho^{\alpha \kappa *} M_{\kappa \beta}\right] .
\end{aligned}
$$

This is the basis for determining $\rho$, this is discussed in Sec. 3. 
As we have performed a canonical transformation it is to be expected that $\tilde{\mathcal{F}}_{i j}$ is real and symmetric in $i$ and $j$, as it holds for $\mathcal{F}_{i j}$. The sum of the first and fourth terms in the bracket can be shown to be real and symmetric in $i$ and $j$ using the relation (A.37), the symmetry of $\rho$ and the Hermiticity of $M$. The sum of the second and third terms in the bracket can be rewritten, using Eq. (A.34) and the symmetry in the summation over $\alpha$ and $\beta$, as

$$
\begin{aligned}
& M_{\alpha \beta} f_{i}^{\alpha} f_{j}^{\beta *}+\rho^{\alpha \lambda} M_{\kappa \lambda} \rho^{\beta \kappa *} f_{i}^{\beta *} f_{j}^{\alpha} \\
& =\delta_{\alpha \beta} f_{i}^{\alpha} f_{j}^{\beta *}+\rho^{\alpha \lambda} M_{\kappa \lambda} \rho^{\beta \kappa *} f_{i}^{\alpha} f_{j}^{\beta *}+\rho^{\alpha \lambda} M_{\kappa \lambda} \rho^{\beta \kappa *} f_{i}^{\beta *} f_{j}^{\alpha} .
\end{aligned}
$$

The first term on the right hand side is the one that appears in the fluctuation integrals $\mathcal{F}_{i j}$. Its sum over $\alpha=\beta$ with prefactor $1 / \Omega_{\alpha 0}$ is real and symmetric in $i$ and $j$, see Eq. (B.8). The sum of the second and third terms on the right hand side is obviously symmetric in $i$ and $j$. It can be shown to be real as well.

The analogy of the various matrices we have defined here with the coefficients obtained in the one-channel case of Ref. [2] is given by $C \leftrightarrow$ $\cosh \gamma, S \leftrightarrow \sinh \gamma \exp (i \delta), \rho \leftrightarrow \tanh \gamma \exp (i \delta), M \leftrightarrow \cosh ^{2} \gamma, M \rho \leftrightarrow$ $\sinh 2 \gamma \exp (i \delta) / 2$, and $2 M-I \leftrightarrow \cosh 2 \gamma$.

\section{B Canonical formalism at $t>0$}

In Sec. 2 we have defined the fluctuation integral

$$
\begin{aligned}
\mathcal{F}_{i j}(t) & =<\psi_{i}(\mathbf{x}, t) \psi_{j}(\mathbf{x}, t)> \\
& =\sum_{\alpha} \int \frac{d^{3} k}{(2 \pi)^{3} 2 \Omega_{\alpha 0}(k)}\left[f_{i}^{\alpha}(k, t) f_{j}^{\alpha *}(k, t)\right] .
\end{aligned}
$$

The expression on the right hand side does not appear to be symmetric in the indices $i$ and $j$, and does not appear to be real. On the other hand the fields $\psi_{i}(\mathbf{x}, t)$ and $\psi_{j}(\mathbf{x}, t)$ should commute with each other. Furthermore, the commutator between $\psi_{i}(\mathbf{x}, t)$ and $\psi_{j}(\mathbf{y}, t)$ is given by the same integral with the only modification that a factor $\exp [i \mathbf{k}(\mathbf{y}-\mathbf{x})]$ appears in the integrand. As these fields commute as well for arbitrary $\mathbf{x}$ and $\mathbf{y}$, the expression

$$
\sum_{\alpha} \frac{1}{2 \Omega_{\alpha 0}(k)} f_{i}^{\alpha}(k, t) f_{j}^{\alpha *}(k, t)
$$


should be real. This is not quite obvious.

To begin with we consider the commutators in $\mathbf{x}$ space. If we calculate the time derivative of the equal time commutator between the fields we get

$$
\frac{d}{d t}\left[\psi_{i}(\mathbf{x}, t), \psi_{j}(\mathbf{y}, t)\right]=\left[\psi_{i}(\mathbf{x}, t), \dot{\psi}_{j}(\mathbf{y}, t)\right]+\left[\dot{\psi}_{i}(\mathbf{x}, t), \psi_{j}(\mathbf{y}, t)\right]
$$

and this is zero if the canonical commutation relations

$$
\left[\psi_{i}(\mathbf{x}, t), \dot{\psi}_{j}(\mathbf{y}, t)\right]=i \delta_{i j} \delta(\mathbf{x}-\mathbf{y})
$$

hold at time $t$. If we require that this relation continues to hold we get the condition

$$
\frac{d}{d t}\left[\psi_{i}(\mathbf{x}, t), \dot{\psi}_{j}(\mathbf{y}, t)\right]=\left[\dot{\psi}_{i}(\mathbf{x}, t), \dot{\psi}_{j}(\mathbf{y}, t)\right]+\left[\psi_{i}(\mathbf{x}, t), \ddot{\psi}_{j}(\mathbf{y}, t)\right]=0 .
$$

The second term can be expressed, using the equation of motion

$$
\ddot{\psi}_{j}-\Delta \psi_{j}+\mathcal{M}_{j k} \psi_{k}=0
$$

by the field commutators; the term vanishes if these commutators take their canonical form at time $t$. We have to require that the first term vanishes:

$$
\left[\dot{\psi}_{i}(\mathbf{x}, t), \dot{\psi}_{j}(\mathbf{y}, t)\right]=0
$$

at time $t$. If this identity shall continue to hold we have to make sure that

$$
\frac{d}{d t}\left[\dot{\psi}_{i}(\mathbf{x}, t), \dot{\psi}_{j}(\mathbf{y}, t)\right]=\left[\dot{\psi}_{i}(\mathbf{x}, t), \ddot{\psi}_{j}(\mathbf{y}, t)\right]+\left[\ddot{\psi}_{i}(\mathbf{x}, t), \dot{\psi}_{j}(\mathbf{y}, t)\right]=0 .
$$

Using again the equation of motion and the symmetry of $\mathcal{M}_{i j}(t)$ this can be verified, if the nontrivial commutations (B.3) hold, whereupon the scheme closes.

This is of course the standard way for proving the time independence of the canonical commutation relations. But this analysis in $\mathbf{x}$ space show us how to proceed in proving the identity

$$
\operatorname{Im} \sum_{\alpha} \frac{1}{\Omega_{\alpha 0}(k)} f_{i}^{\alpha}(k, t) f_{j}^{\alpha *}(k, t)=0
$$


that guarantees the reality and symmetry of the fluctuation integrals. In order for the identity (B.8) to hold at all times, we have to require in addition that the identities

$$
\operatorname{Im} \sum_{\alpha} \frac{1}{\Omega_{\alpha 0}(k)} f_{i}^{\alpha}(k, t) \dot{f}_{j}^{\alpha *}(k, t)=\delta_{i j}
$$

and

$$
\operatorname{Im} \sum_{\alpha} \frac{1}{\Omega_{\alpha 0}(k)} \dot{f}_{i}^{\alpha}(k, t) \dot{f}_{j}^{\alpha *}(k, t)=0
$$

hold independent of time, and that they hold at $t=0$. There is no direct evidence for any of these relations; we just can prove that they continue to hold if they hold at one time. In the one-field case one just has to prove that the canonical commutator is satisfied at all times and that follows from the conservation of the Wronskian. Here the Wronskian of the fluctuations is given, with our initial conditions, by

$$
W\left(f^{\alpha}, f^{\beta}\right)=\sum_{i}\left(f_{i}^{\alpha} \dot{f}_{i}^{\beta *}-\dot{f}_{i}^{\alpha} f_{i}^{\beta *}\right)=2 i \delta_{\alpha \beta} \Omega_{\alpha 0}
$$

where the summation is with respect to the lower indices, while in the commutators we need summations over the upper indices, weighted with $1 / \Omega_{\alpha 0}$.

The proof that the conditions Eqs. (B.8)-(B.10) hold independent of time goes through in analogy to the proof in $\mathbf{x}$ space given above, using this time the equations of motion (2.13). The time derivative (on both sides) of Eq. (B.8) holds, if the relation (B.9) holds. The time derivative of Eq. (B.9) can be shown to hold by using the equations of motion for the fluctuations, and assuming that the relations (B.8) and (B.10) hold. Using again the equations of motion, the time derivative of the relation (B.10) holds if Eq. (B.9) holds.

We still have to consider the initial time $t=0$. With the initial conditions Eqs. (2.14) and (2.15) the relations (B.8) and (B.10) hold trivially as we have chosen the $f_{i}^{\alpha}(0)=f_{i 0}^{\alpha}$ to be the real eigenvectors of the mass matrix. We could still multiply the two eigenvectors with two different phase factors $\exp \left(i \delta_{\alpha}\right)$ without spoiling these conditions. Eq.(B.9) at $t=0$ reduces to

$$
\sum_{\alpha} f_{i 0}^{\alpha} f_{j 0}^{\alpha}=\delta_{i j}
$$

and this is the orthogonality relation dual to

$$
\sum_{i} f_{i 0}^{\alpha} f_{i 0}^{\beta}=\delta_{\alpha \beta}
$$


So at $t=0$ all three relations are satisfied, and then will so for $t>0$.

Aside from their importance for the formalism developed here the relations (B.8)-(B.10) represent useful checks for numerical simulations, along with the time independence of the Wronskian; we have verified this numerically.

\section{References}

[1] V. P. Maslov and O. Y. Shvedov, Theor. Math. Phys. 114, 184 (1998), [hep-th/9709151].

[2] J. Baacke, K. Heitmann and C. Patzold, Phys. Rev. D57, 6398 (1998), [hep-th/9711144].

[3] P. R. Anderson, W. Eaker, S. Habib, C. Molina-Paris and E. Mottola, Int. J. Theor. Phys. 40, 2217 (2001).

[4] K. Goldstein and D. A. Lowe, Phys. Rev. D67, 063502 (2003), [hepth/0208167].

[5] U. H. Danielsson, JHEP 12, 025 (2002), [hep-th/0210058].

[6] C. P. Burgess, J. M. Cline, F. Lemieux and R. Holman, astro$\mathrm{ph} / 0306236$.

[7] K. Schalm, G. Shiu and J. P. van der Schaar, JHEP 04, 076 (2004), [hep-th/0401164].

[8] P. R. Anderson, C. Molina-Paris and E. Mottola, Phys. Rev. D72, 043515 (2005), [hep-th/0504134].

[9] H. Collins and R. Holman, Phys. Rev. D71, 085009 (2005), [hepth/0501158].

[10] H. Collins and R. Holman, Phys. Rev. D74, 045009 (2006), [hepth/0605107].

[11] B. R. Greene, K. Schalm, G. Shiu and J. P. van der Schaar, JCAP 0502, 001 (2005), [hep-th/0411217].

[12] S. Borsanyi and U. Reinosa, 0809.0496. 
[13] J. Baacke, K. Heitmann and C. Patzold, Phys. Rev. D56, 6556 (1997), [hep-ph/9706274].

[14] J. Baacke and C. Patzold, Phys. Rev. D62, 084008 (2000), [hep$\mathrm{ph} / 9912505]$.

[15] E. C. G. Stueckelberg, Phys. Rev. 81, 130 (1951).

[16] N. Bogoliubov and D. Shirkov, Introduction to the Theory of Quantized Fields (Wiley, New York, NY, 1980).

[17] K. Symanzik, Nucl. Phys. B190, 1 (1981).

[18] F. Cooper and E. Mottola, Phys. Rev. D36, 3114 (1987).

[19] J. Baacke, D. Boyanovsky and H. J. de Vega, Phys. Rev. D63, 045023 (2001), [hep-ph/9907337].

[20] J. Baacke, K. Heitmann and C. Patzold, Phys. Rev. D58, 125013 (1998), [hep-ph/9806205].

[21] J. Baacke and C. Patzold, Phys. Rev. D61, 024016 (1999), [hep$\mathrm{ph} / 9906417]$.

[22] J. Baacke and A. Heinen, Phys. Rev. D69, 083523 (2004), [hep$\mathrm{ph} / 0311282]$.

[23] J. Baacke, K. Heitmann and C. Patzold, Phys. Rev. D55, 2320 (1997), [hep-th/9608006].

[24] H. P. Nilles, M. Peloso and L. Sorbo, JHEP 04, 004 (2001), [hepth/0103202].

[25] A. D. Linde, Phys. Rev. D49, 748 (1994), [astro-ph/9307002].

[26] E. J. Copeland, A. R. Liddle, D. H. Lyth, E. D. Stewart and D. Wands, Phys. Rev. D49, 6410 (1994), [astro-ph/9401011].

[27] J. Garcia-Bellido and A. D. Linde, Phys. Rev. D57, 6075 (1998), [hep$\mathrm{ph} / 9711360]$.

[28] K. A. Olive and M. Peloso, Phys. Rev. D74, 103514 (2006), [hep$\mathrm{ph} / 0608096]$. 
[29] R. Allahverdi and A. Mazumdar, JCAP 0708, 023 (2007), [hep$\mathrm{ph} / 0608296]$.

[30] N. Birrell and P. Davies, Quantum fields in curved space (Cambridge University Press, Cambridge, 1982).

[31] J. S. Schwinger, J. Math. Phys. 2, 407 (1961).

[32] L. V. Keldysh, Zh. Eksp. Teor. Fiz. 47, 1515 (1964), [Sov. Phys. JETP 20, 1018 (1965)].

[33] E. Calzetta and B. L. Hu, Phys. Rev. D35, 495 (1987).

[34] R. D. Jordan, Phys. Rev. D33, 444 (1986).

[35] A. Ringwald, Ann. Phys. 177, 129 (1987).

[36] D. Boyanovsky and H. J. de Vega, Phys. Rev. D47, 2343 (1993), [hepth/9211044].

[37] D. Polarski and A. A. Starobinsky, Class. Quant. Grav. 13, 377 (1996), [gr-qc/9504030].

[38] S. Y. Khlebnikov and I. I. Tkachev, Phys. Rev. Lett. 77, 219 (1996), [hep-ph/9603378].

[39] J. Baacke, L. Covi and N. Kevlishvili, work in progress.

[40] N. Bogoliubov and D. Shirkov, Quantum fields (Benjamin/Cummings, Reading, MA, 1983). 